\title{
Behandlung von Patienten mit Asthma bronchiale mit langwirksamen bronchialerweiternden Medikamenten (Formoterol und Salmeterol) Stellungnahme der Deutschen Atemwegsliga und der Deutschen Gesellschaft für Pneumologie und Beatmungsmedizin
}

\author{
Long-Akting Bronchodilators (Formoterol and Salmeterol) for Treatment of Asthma \\ Comment of the German Airway League and the German Respiratory Society
}

\author{
Autor \\ P. Kardos im Auftrag der Deutschen Atemwegsliga und der Deutschen Gesellschaft für Pneumologie \\ und Beatmungsmedizin \\ Institut \\ Gemeinschaftspraxis \& Zentrum für Allergologie, Pneumologie, Schlafmedizin, Klinik Maingau vom Roten Kreuz; \\ Frankfurt am Main
}

\section{Bibliografie}

Dol $10.1055 / \mathrm{s}-0028-1119459$

Pneumologie 2009; 63: 5

(c) Georg Thieme Verlag KG

Stuttgart · New York

ISSN 0934-8387

Korrespondenzadresse

Dr. med. Peter Kardos

Scheffelstr. 33

60318 Frankfurt

kardos@lungenpraxis-

maingau.de
Ein beratendes Fachgremium der US-amerikanischen Zulassungsbehörde FDA (Food and Drug Administration) hat am 11.12.2008 aufgrund einer Metaanalyse klinischer Studien an über 60000 Patienten nach Prüfung des Nutzen/Risikoverhältnisses von der Anwendung lang wirksamer Beta-2-Agonisten (Formoterol und Salmeterol) als Monotherapie bei Asthmapatienten abgeraten. Bereits seit 2006 trugen die in den USA verkauften Packungen eine Warnung vor Nebenwirkungen. Die FDA selbst hat noch keine Entscheidung zum Zulassungsstatus dieser Medikamente getroffen. Reaktionen der Laienpresse (z.B. The New York Times, Wall Street Journal vom 12.12.2008) belegen, dass die Entscheidung in der Öffentlichkeit missverstanden wurde. Auch deutsche Patienten und Ärzte sind erheblich verunsichert.

Die Deutsche Atemwegsliga und die Deutsche Gesellschaft für Pneumologie und Beatmungsmedizin möchten in diesem Zusammenhang die folgenden Punkte klarstellen:

1. Die Empfehlung bezieht sich ausschließlich auf Monopräparate, die Formoterol oder Salmeterol enthalten und nur auf die Indikation Asthma. In deutschen Leitlinien zur Asthmatherapie wird für Erwachsene und Kinder von einer Monotherapie mit diesen Substanzen abgeraten. Beide Präparate wurden in Deutschland für Asthma nur in Verbindung mit einer entzündungshemmenden Dauertherapie (Kortikosteroide) zugelassen. Der Einsatz beider Präparate bei Patienten mit COPD ist nicht berührt.

2. Das FDA-Expertengremium beurteilt das Nutzen/Risikoverhältnis der Kombinationspräparate aus inhalierbaren Kortikosteroiden und beiden langwirksamen Bronchodilatatoren (Formoterol und Salmeterol) bei Asthmapatienten als günstig und riet deshalb nicht zum Verbot der fixen Kombinationen.

Damit hat der Beschluss keine Auswirkungen auf die Therapieempfehlungen für Deutschland.
Die deutsche Ärzteschaft sollte allerdings den adäquaten Einsatz der langwirksamen Beta-2Agonisten Formoterol und Salmeterol überdenken. Die Mehrzahl der Asthmapatienten leidet an leichtem Asthma und benötigt nur niedrig dosierte inhalative Kortikosteroide ohne langwirksame Bronchodilatatoren. Die Verordnung der fixen Kombination und damit eines langwirksamen Bronchodilatators ist bei solchen Patienten nicht notwendig. Ganz besonders gilt diese Aussage für Kinder, bei denen der Nutzen einer Dauertherapie mit einem langwirksamen Bronchodilatator weniger gut gesichert und das Risiko von Nebenwirkungen höher ist als bei Erwachsenen. Für Patienten mit mittel- und schwergradigem Asthma, die von einer Behandlung mit Formoterol bzw. Salmeterol profitieren, sollten die Substanzen in Form fixer Kombinationen mit inhalierbaren Glukokortikoiden verordnet werden. Im direkten Widerspruch zu dieser Empfehlung empfahl das Institut für Qualität und Wirtschaftlichkeit in der Medizin (IQWIG) dem Gemeinsamen Bundesausschuss, die Kosten für derartige fixe Kombinationen für Asthma von der gesetzlichen Krankenversicherung nicht länger erstatten zu lassen. Die Deutsche Atemwegsliga und die Deutsche Gesellschaft für Pneumologie und Beatmungsmedizin haben im IQWIG Anhörungsverfahren ähnliche Bedenken wie jetzt das USamerikanische Expertengremium geäußert (Stellungnahmen 19.6.2007 bzw. 1.7.2008), und fühlen sich durch die jüngste Entscheidung des FDA Expertengremiums vom 11.12.2008 bestätigt. Unsere Argumente blieben vom IQWIG jedoch weitgehend unberücksichtigt. Es bleibt daher zu hoffen, dass der Gemeinsame Bundesausschuss keine Entscheidung treffen wird, die zum vermehrten Einsatz der hierzulande bislang kaum gebräuchlichen Monotherapie für Asthma mit lang wirkenden Beta-2-Agonisten führt und damit unsere Patienten einem unnötigen Risiko aussetzen würde. 